\title{
Versuche zur Darstellung eines Tetraoxydianthrachinonyls mit Alizarinstellung der Hydroxyle
}

von

\section{Christian Seer und Ehrenreich Karl.}

Aus dem chemischen Institut der Universität Graz.

(Vorgelegt in der Sitzung am 9. Jänner 1913.)

I.

Als vor einer Reihe von Jahren durch R. Scholl und seine Mitarbeiter ${ }^{1}$ die für die Darstellung von Küpenfarbstoffen der Anthrachinonreihe wichtige Körperklasse der Dianthrachinonyle erschlossen und als Folge dieser Entdeckung von dem einen von uns auf Veranlassung von $R$. Scholl das 4, 4'-Dioxy$1,1^{\prime}$-dianthrachinonyl ${ }^{2}$ synthetisiert wurde, tauchte auch der Gedarke auf, ein Tetraoxydianthrachinonyl mit Alizarinstellung der Hydroxyle darzustellen, um den Einfluß kennen zu lernen, welchen die Aneinanderkettung zweier Molekeln Alizarin auf die färberischen Eigenschaften des letzteren hervorzurufen vermag.

In der Verfolgung dieser Idee wurde unter anderem auch der vergebliche Versuch angesteltt, das bekannte 4-Aminoalizarin durch Diazotieren und Umsetzung der Diazoniumverbindung mit Kaliumjodid in 4-Jodalizarin zu verwandeln, ${ }^{3}$

I Scholl, B., 40, 1696 (1907).

2 Scholl und Seer, B., 44, 1098 (1911).

3 Diese Versuche wurden im Jahre 1906 an der Technischen Hochschule in Karlsruhe ausgefuhrt. Wie wir jetzt festgestellt haben, läßt sich das 4-Aminoalizarin auch nach der inzwischen von O. N. Witt (B., 42, 2953 [1909]) mitgeteilten Methode zur Diazotierung schwach basischer Amine nicht in das entsprechende Diazoniumsalz verwandeln. 
aus dem wir durch Erhitzen mit Kupferpulver - nötigenfalls nach vorausgegangener Methylierung - das 3,4,3', 4'-Tetraoxy-1, 1'-dianthrachinonyl (I) zu gewinnen hofften. ${ }^{1}$

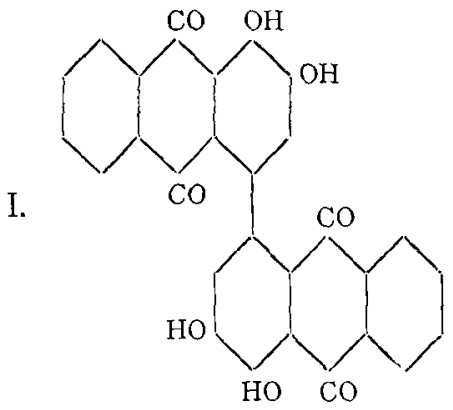

Nachdem inzwischen C. Graebe ${ }^{2}$ die vollständige Methylierung des Alizarins gelungen ist, haben wir die Versuche zur Darstellung des $\gg$ Dializarins (I) wieder aufgenommen.

Durch Nitrieren des Alizarindimethyläthers bei tiefer Temperatur erhielten wir sowohl mit Schwefelsäure und Kaliumnitrat als auch mit Salpetersäure (spez. Gew. 1.51) neben einer geringen Menge eines nicht näher untersuchten Oxykörpers einen Mononitroalizarindimethyläther. $\mathrm{Da}$ beim Nitrieren des Diacetylalizarins unter ähnlichen Bedingungen im wesentlichen eine 4-Nitroverbindung entsteht, ${ }^{3}$ war es von vornherein sehr wahrscheinlich, daß auch in unserem Falle der Eintritt der Nitrogruppe in den Anthrachinonkern an der gleichen Stelle erfolgt, die erhaltene Verbindung also der 4-Nitroalizarindimethyläther war.

Auf folgendem Wege ist es uns gelungen, die Richtigkeit dieser Annahme zu bestätigen: Wir verwandelten die Nitrogruppe durch Reduktion mit Ammoniumsulfid in die Amino-

1 Neuerdings haben wir in Gemeinschaft mit J. Daimer Versuche angestellt, das nach dem D. R. P. 77179 (Farbwerke Meister, Lucius \& Brüning) durch Einleiten von Chlor in eine Lösung von alizarinsulfosaurem Natrium erhältliche 4-Chloralizarin mit Kupferpulver in das Dianthrachinonyl I zu verwandeln, ohne das gewünschte Ziel zu erreichen.

2 Annalen, 349, 201 (1906).

3 Vg1. Brasch, B., 24, 1610 (1891). 
gruppe, ersetzten diese in bekannter Weise durch Jod und erhielten aus dem Jodalizarindimethyläther durch Erhitzen mit Kupferpulver ein Tetramethoxydianthrachinonyl. Dieses zeigt die von Scholl und Mansfeld ${ }^{1}$ gelegentlich der Darstellung von meso-Benzdianthron (III) aus $\alpha$-Dianthrachinonyl (II) entdeckte,

II.

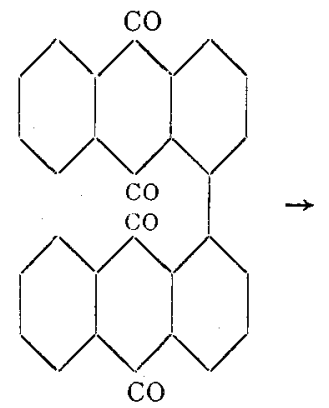

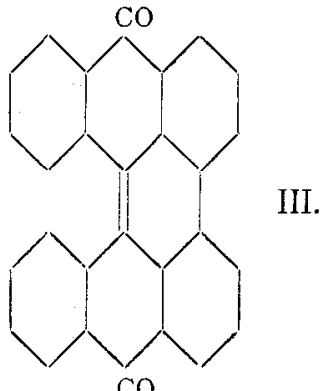

$\mathrm{CO}$

fïr a-Dianthrachinonyle charakteristische Reaktion mit Kupferpulver und konzentrierter Schwefelsäure. Die Lösung der Substanz in konzentrierter Schwefelsäure ist kirschrot und wird auf Zusatz von etwas Kupferpulver und kurzem, sehr schwachem Erwärmen dunkelgrün, ${ }^{2}$ unter Bildung von $3,4,3^{\prime}, 4^{\prime}$ Tetramethoxy-meso-benzdianthron (VI). Da demnach der vorliegende Körper das 3,4,3', $4^{\prime}$-Tetramethoxy-1, $1^{\prime}$-dianthrachinonyl $(V)$ ist, muß beim Nitrieren des Alizarindimethyläthers - wie zu erwarten war - der 4-Nitroalizarindimethyläther (IV) entstanden sein.

Durch Erhitzen des 3, 4, 3', $4^{\prime}$-Tetramethoxy-1, 1'- dianthrachinonyls (V) mit Aluminiumchlorid hofften wir, das gesuchte "Dializarin (I) zu erhaiten; es zeigte sich jedoch, daß hierbei nur zwei Methoxylgruppen verseift werden. Aus Analogiegründen muß man schließen, daß hier ebenso wie beim Alizarindimethyläther ${ }^{3}$ die $\alpha$-ständigen Methoxyle verseift werden und

1 B., 43, 1734 (1910).

2 Die rote Lösungsfarbe von Alizarin in konzentrierter Schwefelsäure wird durch Kupferpulver auch in der Wärme nicht verändert.

3 Nach Graebe (Annalen, 349, 203 [1906]) liefert Alizarindimethyläther mit Alkalien bei $130^{\circ}$ und mit konzentrierter Bromwasserstoffsäure den 2-Methylester des Alizarins. 
demnach das 4, $4^{\prime}$-Dioxy-3, $3^{\prime}$-dimethoxy-1,1'-dianthrachinonyl (VII) entstanden ist.

IV.<smiles>COC1C2CC3CCCCC2C(=O)C3C1[N+](=O)[O-]</smiles>
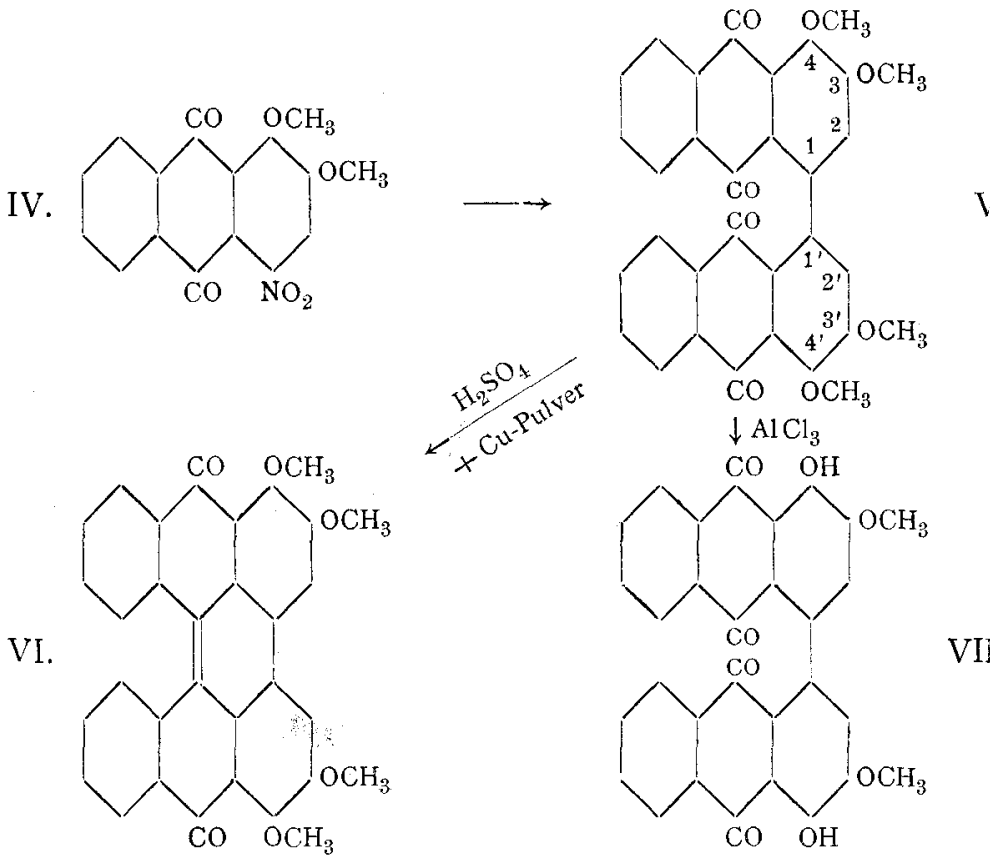

$V$.

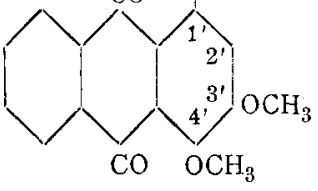

$\downarrow \mathrm{AlCl}_{3}$

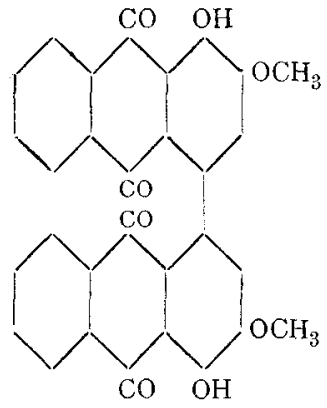

VII.

II.

Da der aben beschriebene Weg zur Synthese des $3,4,3^{\prime}, 4^{\prime}$ Tetraoxy-1,1'-dianthrachinonyls nicht zum Ziele führte, haben wir noch versucht, zum Aufbau dieses Körpers die von Scholl und See ${ }^{1}$ zur Darstellung von Oxydianthrachinonylen benutzte Backmethode anzuwenden. Nach dieser entsteht $z$. B. beim Zusammenbacken von 4, $4^{\prime}$-Dioxy-1, $1^{\prime}$-biphenyl oder 4, 4'-Dimethoxy-1, 1'-biphenyl mit Phthalsäureanhydrid und Aluminiumchlorid neben verschiedenen anderen Produkten in ziemlich guter Ausbeute 4, 4'-Dioxy -1, 1'-dianthrachinony1.

Als Ausgangsprodukt benötigten wir dazu das $3,4,3^{\prime}, 4^{\prime}$ Tetraoxy-1, 1'-biphenyl (Dibrenzkatechin) oder das $3,4,3^{\prime}, 4^{\prime}$ Tetramethoxy-1, $1^{\prime}$-biphenyl (Diveratryl). Das erstere wurde

1 B., 44, 1098 (1911). 
bereits von Barth und Schreder ${ }^{1}$ durch Alkalischmelze der $o$-Biphenyldisulfonsäure erhalten und wird von diesen Autoren als ein sehr luftempfindlicher und zersetzlicher Körper geschildert, der nur durch Sublimation im Wasserstoffstrom in kleiner Menge rein erhalten werden konnte. Wir entschlossen uns deshalb, das Diveratryl (VIII) zum Aufbau des gesuchten "Dializarins « zu verwenden,
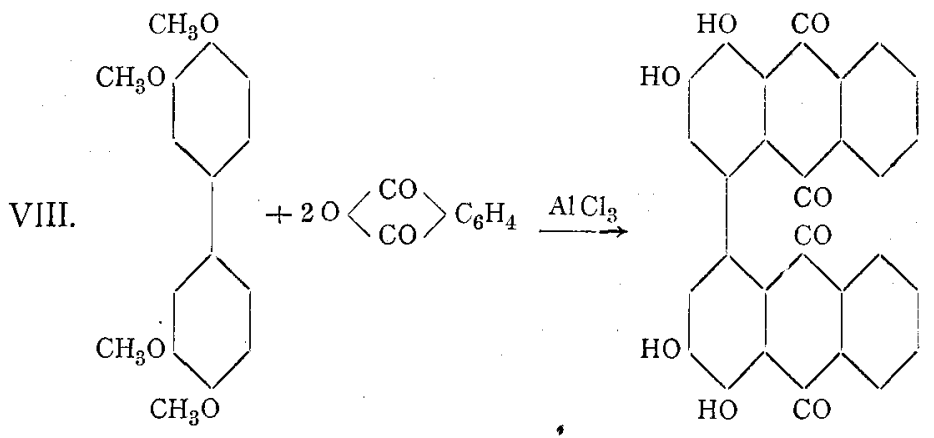

und stellten Versuche an, diese bis dahin unbekannte Verbindung darzustellen. Dabei stießen wir zunächst auf unvorhergesehene Schwierigkeiten.

4-Jodveratrol, das sich nach der Ullmann'schen Methode mit Kupferpulver in das gesuchte Diveratryl (VIII) verwandeln lassen mußte, ist schon von Tassilly und Leroide ${ }^{2}$ durch Methylieren von 4-Jodguajakol erhalten worden; dieser Weg ist aber, wie wir uns durch einige Versuche überzeugten, zur Gewinnung größerer Mengen dieses Körpers nicht geeignet. Das gegebene Ausgangsprodukt zu seiner Darstellung ist das Veratrol, welches beim Nitrieren in fast quantitativer Ausbeute 4 -Nitroveratrol liefert, ${ }^{3}$ das bereits von $\mathrm{Moureu}{ }^{4}$ durch Reduktion mit Zinn und Salzsäure in 4-Veratrylamin übergeführt wurde. Dieses versuchten wir über die Diazoniumverbindung in 4-Jodveratrol $\mathrm{zu}$ verwandeln. ${ }^{5}$ Dabei gelang es

1 B., 11,1336 (1878).

2 C. r., 144, 757; C. 1907 , II, 46.

s. Merk, Annalen, 108, 60 (1858).

4 C. r., 122, 477 (1896).

5 Tassi11y und Leroide haben auf diesem Wege schon cas 4-Jodveratrol erhalten. Da das Zentralblatt sich aber mit der bloßen Mitteilung dieser 
unș zunächst nicht, den Jodkörper in reiner Form zu gewinnen. Da wir jedoch der festen Überzeugung waren, im wesentlichen das richtige Produkt in Händen zu haben, erbitzten wir den unreinen Jodkörper mit Kupferpulver und gewannen auf diese Weise einen in farblosen Nadeln krystallisierenden Körper von konstantem Schmelzpunkt. $\mathrm{Zu}$ unserer großen Überraschung ergab die Elementaranalyse dieser Substanz etwa $15 \%$ zu wenig Kohlenstoff für das erwartete Tetramethoxybiphenyl, und die nähere Untersuchung zeigte, daß der Körper zwar kein Jod mehr, wohl aber zwei Atome Chlor enthielt. Wie sich weiter herausstellte, war das Chlor bereits bei der Reduktion des Nitroveratrols mit Zinn und Salzsäure eingetreten, so daß wir nicht das gewünschte Veratrylamin, sondern ein Chlorveratrylamin erhalten hatten. Man darf wohl mit einiger Wahrscheinlichkeit annehmen, daß das Chlor paraständig zu einer Methoxygruppe eingetreten und ein 5-Chlor-4-veratrylamin (IX)

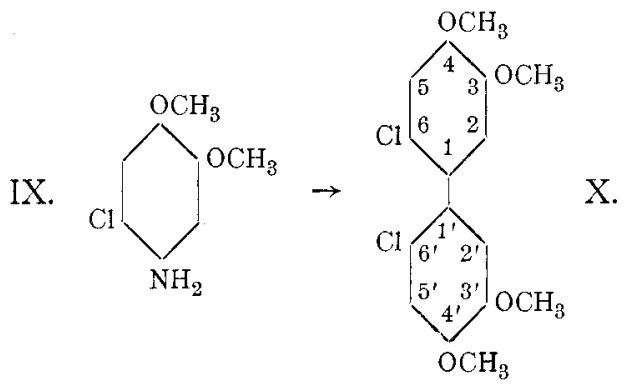

entstanden ist, das bei der oben beschriebenen weiteren Behandlung in 6, 6'-Dichlor-3, 4, 3', $4^{\prime}$-tetramethoxy-1, $1^{\prime}$-biphenyl (X) verwandelt wurde. Wie wir weiter feststellen konnten, erhält man fast ausschließlich die Chlorverbindung IX, wenn man die Reduktion des 4-Nitroveratrols in der Hitze vornimmt. Nachdem wir diese Verbindung isoliert und gereinigt hatten, gelang. es auch, das 4-Jod-5-chlorveratrol in reiner Form zu gewinnen.

Tatsache begnügt (C., 1907, II, 976) und uns die Originalarbeit (Bull. des Sciences Pharmacol., 14, 400) nicht zur Verfügung stand, waren wir gezwungen, eigene Versuche anzustellen. 
Der Eintritt von Chlor in den Kern bei der Reduktion aromatischer Nitrokörper mit Zinn und Salzsäure ist schon einige Male beobachtet worden; ${ }^{1}$ so liefert $z$. B. nach Jann asch ${ }^{2}$ 2 -Nitro- $p$-xylol $50 \%$ der chlorfreien und $50 \%$ einer gechlorten Base:

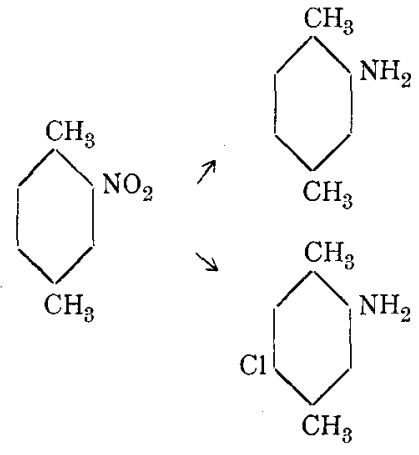

Führt man die Reduktion des 4-Nitroveratrols unter möglichst gemäßigten Bedingungen aus - wir verfuhren in der Weise, daß wir den Nitrokörper in konzentrierter Salzsäure suspendierten, das Zinn allmählich eintrugen und eine zu starke Erwärmung durch Außenkühlung mit fließendem Wasser verhinderten -, so entsteht nach Versuchen, die der eine von uns in Gemeinschaft mit Herrn J. Daimer ausführte, die von Moureu (1. c.) beschriebene chlorfreie Base, die wir dann über das 4-Jodveratrol in das gesuchte $3,4,3^{\prime}, 4^{\prime}$-Tetramethoxy$1,1^{\prime}$-biphenyl (VIII) verwandeln konnten. Die Gewinnung größerer Mengen des Körpers ist jedoch auf diesem Wege ziemlich mühsam, und wir suchten deshalb nach einer bequemeren Darstellung des 4-Jodveratrols, die uns auch durch direkte Jodierung von Veratrol bei Gegenwart von Quecksilberoxyd gelang.

Das auf diesem Wege erhaltene Jodveratrol erwies sich als identisch mit dem aus 4-Veratrylamin dargestellten; beide Körper gaben beim Erhitzen mit Kupferpulver das gleiche Diveratryl.

1 Zusammenstellung der Literatur in Weyl's Methoden der organischen Chemie, Bd. II, p. 210.

2 Annalen, 156, 81 (1870). 
Obwohl dieses nun verhältnismäßig mühelos zu gewinnen war, haben wir doch das oben erwähnte Endziel nicht erreichen können. $3,4,3^{\prime}, 4^{\prime}$-Tetramethoxy-1,1'-biphenyl tritt bei der Friedel-Crafts'schen Reaktion bei Gegenwart von Lösungsmitteln (Schwefelkohlenstoff oder Nitrobenzol) mit Phthalsäureanhydrid nicht in Reaktion.

Beim Zusammenbacken des Körpers mit Phthalsäureanhydrid und Aluminiumchlorid erhielten wir ein dunkelblaues Reaktionsprodukt, in dem wahrscheinlich unter anderen Körpern auch das gesuchte "Dializarin « enthalten war; es ist uns jedoch nicht gelungen, dieses in reiner Form $\mathrm{zu}$ isolieren.

\section{Experimenteller Teil.}

\section{I.}

4-Nitroalizarindimethyläther (Formel IV).

a) Durch Nitrierung von Alizarindimethyläther mittels Kaliumnitrat und konzentrierter Schwefelsäure.

$1.3 \mathrm{~g}$ Alizarindimethyläther werden in $20 \mathrm{~cm}^{3}$ konzentrierter Schwefelsäure gelöst, die Lösung auf $0^{\circ}$ abgekühlt und $0.6 \mathrm{~g}$ pulverisiertes Kaliumnitrat unter Umschütteln allmählich eingetragen, so daß die Temperatur $0^{\circ}$ nicht übersteigt. Nach 20 stündigem Stehen gießt man die dunkelrote Lösung unter Turbinieren in Wasser ein, wobei sich der gebildete Nitrokörper, der noch durch ein alkalilösliches Nebenprodukt verunreinigt ist, in orangeroten Flocken ausscheidet. Diese werden abfiltriert und zur Entfernung der Schwefelsäure mit Wasser gründlich ausgewaschen. Das getrocknete Rohprodukt wird darauf mit wenig kaltem Chloroform behandelt, wobei die Hauptmenge des Nebenproduktes (Schmelzpunkt unscharf 270 bis $290^{\circ}$ ) ungelöst bleibt. Das Filtrat wird heiß mit Methylalkohol versetzt, bis sich der Nitrokörper in orangegelben Krystallen auszuscheiden beginnt. Diese werden durch weiteres Umkrystallisieren aus Eisessig gereinigt.

Etwas umständlicher, aber weniger verlustreich ist folgender Weg zur Reinigung: Das rohe, mit Wasser gut ausgewaschene Nitrierungsprodukt wird dreimal aus Eisessig 
umkrystallisiert. Dadurch erhält man einen Teil des Nitrokörpers rein in Form dunkelgelber, derber, prismatischer Nadeln vom Schmelzpunkt 209 bis $210^{\circ}$. Die Eisessigmutterlaugen werden in Wasser gegossen, die ausgeschiedenen Flocken abfiltriert, getrocknet und in Benzol gelöst. Die benzolische Lösung wird mit sehr verdïnnter Natronlauge (der Oxykörper bildet ein schwer lösliches Natriumsalz) so lange durchgeschüttelt, bis die Lauge nicht mehr rot gefärbt wird. Durch Abdunsten des Benzols und Umkrystallisieren des Rückstandes aus Eisessig erhält man eine weitere Menge von reinem 4-Nitroalizarindimethyläther. Die Gesamtausbeute an krystallisiertem Nitrokörper beträgt $1.3 g$, d. i. $86 \%$ der Theorie.

$0 \cdot 2217 \mathrm{~g}$ Substanz: $9 \cdot 2 \mathrm{~cm}^{3} \mathrm{~N}\left(744 \mathrm{~mm}, 23^{\circ}\right)$.

In 100 Teilen:

$$
\mathrm{N} \ldots \ldots \ldots \ldots \underbrace{\text { Gefunden }}_{4 \cdot 68} \quad \underbrace{\begin{array}{c}
\text { Berechnet für } \\
\mathrm{C}_{16} \mathrm{H}_{11} \mathrm{O}_{6} \mathrm{~N}
\end{array}}_{4 \cdot 49}
$$

4-Nitroalizarindimethyläther ist sehr schwer löslich in Alkohol, Äther und Schwefelkohlenstoff, leicht löslich in heißem Aceton, Eisessig, Nitrobenzol und in Chloroform schon in der Kälte. Die Lösungsfarbe in konzentrierter Schwefelsäure ist rot. Alkalisches Natriumhydrosulfit löst zunächst wahrscheinlich infolge Reduktion der Nitrogruppe (vgl. unten die Lösungsfarbe des Aminoalizarindimethyläthers) mit violetter Farbe, die beim Erhitzen in Rot umschlägt. Dieser Farbenwechsel dürfte entweder durch eine teilweise Verseifung der Methoxygruppen oder durch den Ersatz der zunächst durch Reduktion entstandenen Aminogruppe durch Hydroxyl hervorgerufen werden.

\section{b) Mit konzentrierter Salpetersäure.}

Bei vorsichtigem Nitrieren von Alizarindimethyläther mit Salpetersäure (spez. Gew. 1.51) unter $0^{\circ}$ erhält man ebenfalls im wesentlichen Mononitroalizarindimethyläther; als Nebenprodukt entsteht auch hierbei ein roter, in Alkalien döslicher Körper.

Der Alizarindimethyläther verhält sich also beim Nitrieren ähnlich wie das Diacetylalizarin und Alizarin selbst, bei denen 
als Nebenprodukte Nitrooxyalizarin und Purpurin aufgefunden wurden. ${ }^{1}$

\section{4-Aminoalizarindimethyläther}<smiles>COC1C(N)C2C(=O)C3CCCCC3CC2C1OC</smiles>

erhält man durch einstündiges Turbinieren des amorphen Nitrokörpers mit wässerigem Ammoniumsulfid bei Wasserbadtemperatur. Nach dem Erkalten wird der dunkelrote Aminokörper abgesaugt, auf dem Filter mit Wasser gewaschen, bis das Filtrat farblos durchläuft, dann getrocknet, gepulvert und zur Entfernung von ausgeschiedenem Schwefel in Schwefelkohlenstoff suspendiert. Nach mehrstündigem Stehen wird der Niederschlag abgesaugt und auf dem Filter mit Schwefelkohlenstoff und Äther gewaschen, Durch Umkrystallisieren des Rohproduktes aus verdünntem Pyridin erhält man dunkelrote lanzettförmige Kryställchen, die zuweilen grünen Oberflächenglanz zeigen. Der Schmelzpunkt der Substanz ist infolge der dunklen Körperfarbe schwer zu beobachten; er liegt nach einmaligem Umkrystallisieren etwa bei 182 bis $185^{\circ}$. Ausbeute $67 \%$ der Theorie.

$0 \cdot 1188 g$ Substanz: $0.2948 g \mathrm{CO}_{2}, 0.0548 g \mathrm{H}_{2} \mathrm{O}$.

$0 \cdot 2751 \mathrm{~g}$ Substanz: $11 \cdot 6 \mathrm{~cm}^{3} \mathrm{~N}\left(25^{\circ}, 740 \mathrm{~mm}\right)$.

In 100 Teilen:

\begin{tabular}{|c|c|c|}
\hline & Gefunden & $\begin{array}{l}\text { Berechnet fiù } \\
\mathrm{C}_{16} \mathrm{H}_{18} \mathrm{O}_{4} \mathrm{~N}\end{array}$ \\
\hline C. & $67 \cdot 68$ & $67 \cdot 82$ \\
\hline . & $5 \cdot 16$ & $4 \cdot 63$ \\
\hline 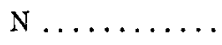 & $4 \cdot 81$ & $4 \cdot 96$ \\
\hline
\end{tabular}

4-Aminoalizarindimethyläther ist sehr schwer löslich in Alkohol, Äther, Ligroin und Schwefelkohlenstoff, leicht löslich in den übrigen organischen Lösungsmitteln; er krystallisiert

1 Vgl. R. Brasch, B., 24, 1610 (1891); daselbst Zusammenstellung der Literatur. 
- jedoch nur dann, wenn aus dem Rohprodukt der beigemengte Schwefel möglichst entfernt ist - aus verdünntem Pyridin, Eisessig oder Methylalkohol.

Bei längerem Kochen mit verdünnter Natronlauge nimmt diese allmählich eine violette Färbung an. ${ }^{1}$ Wahrscheinlich dürfte dabei an Stelle der Aminogruppe ein Hydroxyl treten, denn gegen eine Verseifung der Methoxylgruppen spricht das Verhalten des 4-Jodalizarindimethyläthers und des $3,4,3^{\prime}, 4^{\prime}$ Tetramethoxy-1,1'- dianthrachinonyls (vgl. unten), die durch siedende Natronlauge nicht angegriffen werden.

Mit alkalischem Hydrosulfit entsteht zunächst eine violette Lösung, die später in Rot umschlägt (vgl. oben das Verhalten des Nitrokörpers).

In konzentrierter Schwefelsäure löst sich der Aminokörper mit blutroter Farbe.

\section{4-Jodalizarindimethyläther.}

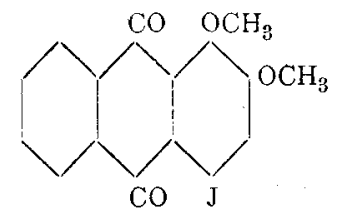

Eine Lösung von $4.5 \mathrm{~g}$ 4-Aminoalizarindimethyläther in Eisessig wird mit $20 \mathrm{~cm}^{3} 1 / 10$ normaler Salzsäure und $100 \mathrm{~cm}^{3}$. Wasser (man verwendet so viel Eisessig, daß sich kein Chlorhydrat ausscheidet) versetzt und bei $0^{\circ}$ unter Turbinieren eine wässerige Lösung von $1.13 \mathrm{~g}$ Natriumnitrit eingetropft. Die lichtrote Farbe der Lösung schlägt dabei in Gelb um. Nach beendigter Diazotierung filtriert man die Diazoniumlösung in eine verdünnte wässerige Lösung von $6 \cdot 3 g$ Kaliumjodid. Auf dem Fitter bleibt eine geringe Menge eines bei der Reaktion gebildeten Oxykörpers zurück. Das Diazoniumjodid wird nach mehrstündigem Stehen durch Einleiten von Wasserdampf verkocht, wodurch gleichzeitig freies Jod entfernt wird. Die Aus-

1 Verwendet man bei der Redulktion des Nitrokörpers anstatt Ammonsulfid Natriumsulfid, so erhält man nur $43 \%$ der Theorie an rohem Aminokörper; der Rest ist alkatilöslich geworden. 
beute an Rohprodukt betrug $4.8 g$, d. i. $76.9 \%$ der Theorie. Durch Umkrystallisieren desselben aus Eisessig erhält man gelbbraune prismatische Nadeln, deren Schmelzpunkt nach dreimaliger Krystallisation bei 172 bis $174^{\circ}$ liegt.

$0 \cdot 1120 \mathrm{~g}$ Substanz: $0.0672 \mathrm{~g} \mathrm{AgJ}$.

In 100 Teilen:

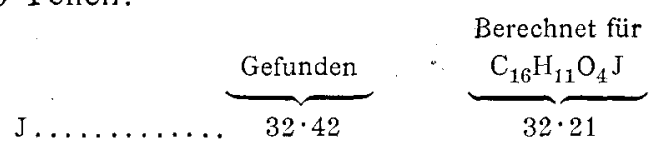

4-Jodalizarindimethyläther ist ziemlich schwer löslich in Alkohol, leicht löslich in Benzol, Eisessig und Chloroform. Die Lösungsfarbe in konzentrierter Schwefelsäure ist dunkelrot. Mit alkalischem Natriumhydrosulfit gibt er eine rote küpenartige Lösung. Durch siedende Alkalien wird er im Gegensatz zum Nitro- und Aminokörper nicht angegriffen,

\section{$3,4,3^{\prime}, 4^{\prime}$-Tetramethoxy-1, $1^{\prime}$-dianthrachinonyl (Formel V).}

Ein inniges Gemisch gleicher Gewichtsteile 4-Jodalizarindimethyläther und Kupferpulver wird im Kohlendioxydstrom im Verlauf einer halben Stunde auf $310^{\circ}$ erhitzt und die Temperatur des Bades dann noch 10 Minuten auf gleicher Höhe erhalten. Die erkaltete Schmelze wird fein gepulvert, mit 15 Gewichtsteilen Benzol übergossen und 24 Stunden sich selbst überlassen. Darauf filtriert man den Niederschlag ab und wäscht mit kaltem Benzol nach. (Das Benzol hinterläßt beim Abdampfen braune Schmieren.) Der Rückstand wird mit sehr viel Xylol mehrmals ausgekocht und heiß filtriert. Beim Erkalten scheidet sich das Dianthrachinonyl im Filtrat krystallinisch aus. Ausbeute $53 \%$ der Theorie. Zur vollständigen Reinigung wird die Substanz in Chloroform gelöst, von einigen ungelösten braunen Flocken abfiltriert und das Filtrat bis zur beginnenden Krystallabscheidung mit heißem Alkohol versetzt. Die abfiltrierten Krystalle werden dann nochmals aus Nitrobenzol umkrystallisiert. Man erhält so goldglänzende mikroskopische Blättchen von rhomboedrischer Form (Schmelzpunkt 
337 bis $338^{\circ}$ ), die für die Analyse mit Alkohol und Äther gewaschen und bei $195^{\circ}$ getrocknet wurden.

$0 \cdot 1192 g$ Substanz: $0.3128 g \mathrm{CO}_{2}, 0.0450 \mathrm{~g} \mathrm{H}_{2} \mathrm{O}$.

In 100 Teilen:

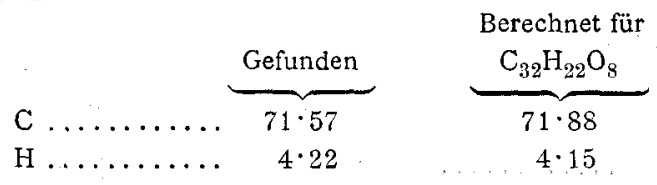

$3,4,3^{\prime}, 4^{\prime}$-Tetramethoxy-1, $1^{\prime}$ - dianthrachinonyl ist in den tiefer siedenden Lösungsmitteln, wie Alkohol, Benzol usw. so gut wie unlöslich, sehr wenig löslich in Eisessig; schwer löslich in Xylol (etwa $0.5 \mathrm{~g}$ im Liter), leicht löslich dagegen in Nitrobenzol und Chloroform. In konzentrierter Schwefelsäure löst es sich mit kirschroter Farbe, die nach Zusatz von Kupferpulver und kurzem, sehr schwachem Erwärmen in Dunkelgrün umschlägt. Von Alkalien und Ammoniak wird es auch bei längerem Kochen nicht angegriffen. Mit alkalischem Natriumhydrosulfit gibt es eine durchsichtig rote küpenartige Lösung, welche ungebeizte Baumwolle nicht anfärbt.

\section{4, 4'-Dioxy-3, 3'-dimethoxy-1, 1'-dianthrachinonyl (Formel VII).}

$\lg 3,4,3^{\prime}, 4^{\prime}$-Tetramethoxy -1, $1^{\prime}$ - dianthrachinonyl wird mit $10 \mathrm{~g}$ wasserfreiem Aluminiumchlorid innig vermischt und unter Chlorcalciumverschluß 3 Stunden auf 150 bis $160^{\circ}$ erhitzt. Nach dem Erkalten wird die violettbraune Schmelze mit verdünnter Salzsäure zersetzt, einige Zeit zum Sieden erhitzt, abfiltriert und ausgewaschen. Das so von Aluminium befreite Reaktionsprodukt wird mit stark verdünnter Natronlauge gekocht, die blauviolette Lösung heiß abfiltriert (beim Erkalten. scheidet sich das Natriumsalz aus) und der in Lösung befindliche Oxykörper im Filtrat mit Salzsäure gefällt. Die ausgeschiedenen rostbraunen Flocken $(0 \cdot 6 g)$ werden abfiltriert, mit.Wasser gut ausgewaschen, getrocknet und aus Nitrobenzol umkrystallisiert. Man erhält sternförmig angeordnete, orangerote, mikroskopische Prismen, die für die Analyse mit Alkohol und 
Äther gewaschen und bei $200^{\circ}$ getrocknet wurden. Die Substanz sublimiert oberhalb $320^{\circ}$ unter allmählichem Zusammensintern und ist bei $350^{\circ}$ noch nicht vollständig geschmolzen.

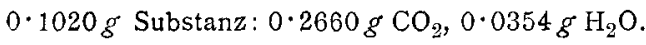

In 100 Teilen:

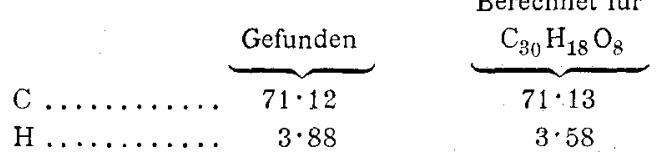

$4,4^{\prime}$-Dioxy-3, $3^{\prime}$-dimethoxy-1, $1^{\prime}$-dianthrachinonyl ist in den meisten organischen Lösungsmitteln praktisch unlöslich, sehr schwer löslich in heißem Chloroform und Nitrobenzol, etwas leichter löslich in Chinolin und Pyridin. Alkalien und Ammoniak lösen es mit blauvioletter Farbe (ähnlich der Lösungsfarbe des Alizarins, nur etwas blaustichiger), die auf Zusatz von Natriumhydrosulfit in Rot umschlägt. Weder ungebeizte noch gebeizte Baumwolle wird nach den üblichen Verfahren angefärbt; löst man dagegen den Oxykörper in Pyridin, fügt Wasser hinzu und kocht mit der verdünnten Pyridinlösung Scheurerstreifen, so werden diese in Tönen angefärbt, welche den Alizarinlacken ähnlich, aber weniger intensiv und leuchtend sind.

Von kalter konzentrierter Schwefelsäure wird die Substanz nur schwer angegriffen; beim Erwärmen entsteht eine intensiv rote Lösung, die beim fast beginnenden Sieden der Schwefelsäure in Blau umschlägt.

\section{II.}

5(?)-Chlor-4-veratrylamin (Formel IX).

Man suspendiert $16 \mathrm{~g}$ 4-Nitroveratrol in $200 \mathrm{~cm}^{3}$ konzentrierter Salzsäure, fügt $16 \mathrm{~g}$ granuliertes $Z$ inn hinzu und erwärmt das Ganze in einem mit Steigrohr versehenen Kolben eine halbe Stunde lang auf einem siedenden Wasserbade. Die Reaktion verläuft sehr stürmisch unter starker Wärmeentwicklung und gibt sich außerdem durch einen Farbenumschlag von Gelb in Lila $z$ u erkennen. Nach dem Erkalten filtriert man das 
ausgeschiedene Zinndoppelsalz ab, löst dieses in viel Wasser und entzinnt mit Schwefelwasserstoff. Das Filtrat des Zinnsulfides wird auf dem Wasserbad unter fortwährendem Durchleiten von Schwefelwasserstoffgas bis auf ein kleines Volumen eingeengt; auf Zusatz von konzentrierter Salzsäure scheidet sich dann das Chlorhydrat der Base in oberflächlich violett gefärbten Krystallen aus. Durch Umkrystallisieren derselben aus Methylalkohol unter Verwendung von Tierkohle erhält man farblose Nadeln, die keinen bestimmten Schmelzpunkt zeigen, sondern sich oberhalb $150^{\circ}$ allmählich zersetzen.

Die wässerige Lösung des Chlorhydrates gibt auf Zusatz von Ferrichlorid eine blaue Färbung, die allmählich in Grün und schließlich in Braun übergeht.

Fügt man $z u$ der verdünnten wässerigen Lösung des Sulfates einige Tropfen Kaliumbichromat, so entsteht zunächst eine grüne Färbung, die später in Gelb umschlägt. ${ }^{1}$

Die freie Base erhält man durch Zersetzen des Chlorhydrates mit Ammoniak, Ausäthern der öligen Suspension und Eindampfen der mit Kaliumcarbonat getrockneten Ätherlösung im Vakuum in Form von farblosen Nadeln (Schmelzpunkt 72 bis $73^{\circ}$ ).

$0.1278 \mathrm{~g}$ Substanz: 0.0962 $\mathrm{g} \mathrm{AgC1}$.

In 100 Teilen:

$$
\mathrm{Cl} \ldots \ldots \ldots \ldots \underbrace{\text { Gefunden }}_{18.63} \quad \overbrace{18: 91}^{\begin{array}{c}
\text { Berechnet für } \\
\mathrm{C}_{8} \mathrm{H}_{10} \mathrm{O}_{2} \mathrm{NCl}
\end{array}}
$$

5-Chlor-4-veratrylamin ist sehr leicht löslich in den meisten organischen Lösungsmitteln, schwer löslich in Ligroin und ziemlich löslich in siedendem Wasser. Aus Wasser und Ligroin krystallisiert es in farblosen Nadeln.

\section{5(?)-Chlor-4-jodveratrol.}

$1.5 \mathrm{~g} \mathrm{5-Chlor-4-veratrylamin} \mathrm{werden} \mathrm{in} \mathrm{überschüssiger}$ verdünnter Salzsäure suspendiert und unter guter Kühlung mit einer wässerigen Lösung von $0.6 \mathrm{~g}$ Natriumnitrit diazotiert.

1 Reaktionen auf Veratrylamin siehe P. Ja cobson, B., 29, 2690 (1896). 
Die filtrierte Diazoniumlösung läßt man in eine gekühlte Lösung von $3 g$ Kaliumjodid einlaufen, erwärmt nach einigen Stunden kurze Zeit auf dem Wasserbad und entfernt ausgeschiedenes Jod durch Behandeln mit Natriumbisulfitlauge. Das gebildete 5-Chlor-4-jodveratrol bleibt als braunes Öl zurück, das nach einiger Zeit erstarrt. Die weitere Reinigung geschieht durch Umkrystallisieren aus verdünntem Methylalkohol unter Verwendung von Tierkohle. Man erhält so farblose Nädelchen vom Schmelzpunkte 69 bis $70^{\circ}$.

$0 \cdot 1255 g$ Substanz: $0 \cdot 1596 g$ AgCl$+\mathrm{AgJ}$.

In 100 Teilen:

$$
\mathrm{AgCl}+\mathrm{AgJ} \ldots \ldots \underbrace{\text { Gefunden }}_{127 \cdot 17} \quad \underbrace{\begin{array}{c}
\text { Berechnet für } \\
\mathrm{C}_{8} \mathrm{H}_{8} \mathrm{O}_{2} \mathrm{C} 1 \mathrm{~J}
\end{array}}_{126 \cdot 74}
$$

5-Chlor-4-jodveratrol löst sich in konzentrierter Schwefelsäure mit violetter Farbe, die bei gewöhnlicher Temperatur allmählich, bei gelindem Erwärmen schnell in Grün übergeht; beim Erhitzen bis zum Sieden der Schwefelsäure findet unter Entwicklung von Schwefeldioxyd ein weiterer Farbenumschlag nach Rotbraun statt.

\section{6, 6' (?)-Dichlor-3, 4, 3', 4'-tetramethoxy -1,1'- bipheny1}

(Formel X).

Ein Gemisch von 1 Teil 5-Chlor-4-jodveratrol mit 1 Teil Kupferpulver wird in einem mit Steigrohr versehenen Gefäße unter Durchleiten von Kohlendioxyd im Verlauf einer halben Stunde bis $270^{\circ}$ und dann noch 10 Minuten auf $280^{\circ}$ erhitzt. Nach dem Erkalten wird die Schmelze gepulvert und der Sublimation im Kohlendioxydstrom unterworfen. Durch zweimaliges Umkrystallisieren des Sublimates aus Methylalkohol erhält man farblose Nädelchen vom Schmelzpunkt 160 bis $161^{\circ}$. Die Ausbeute an reiner Substanz betrug $0.7 g$ aus $6 g$ unreinem Jodkörper.

$0.1082 \mathrm{~g}$ Substanz: $0.2229 \mathrm{~g} \mathrm{CO}_{2}, 0.0473 \mathrm{~g} \mathrm{H}_{2} \mathrm{O}$.

$0 \cdot 1110 \mathrm{~g}$ Substanz: $0 \cdot 2263 g \mathrm{CO}_{2}, 0.0531 \mathrm{~g} \mathrm{H}_{2} \mathrm{O}$.

$0.1134 \mathrm{~g}$ Substanz: $0.0957 \mathrm{~g} \mathrm{AgCl}$. 
In 100 Teilen:

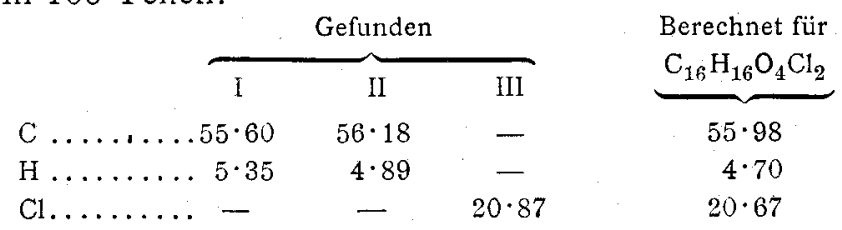

6, $6^{\prime}$-Dichlor-3, 4, $3^{\prime}, 4^{\prime}$-tetramethoxy-1, $1^{\prime}$-biphenyl ist sehr leicht löslich in Ligroin, Benzol, Eisessig, Aceton und Äther, etwas schwerer löslich in Methylalkohol. Von konzentrierter Schwefelsäure wird die krystallisierte Substanz in der Kälte schwer angegriffen; bei gelindem Erwärmen löst sie sich mit schwach grüner Farbe, die bei höherer Temperatur intensiv dunkelgrün wird.

\section{4-Jodveratrol, $\mathrm{C}_{6} \mathrm{H}_{3} . \mathrm{OCH}_{3}(1) . \mathrm{OCH}_{3}(2) . \mathrm{J}(4)$.}

$20 \mathrm{~g}$ Veratrol werden in $30 \mathrm{~g}$ Alkohol gelöst, in die Lösung $20 \mathrm{~g}$ Jod und $16 \mathrm{~g}$ Quecksilberoxyd portionsweise eingetragen und das Ganze 4 Stunden auf der Schüttelmaschine geschüttelt. Hierauf filtriert man, destilliert vom Filtrat den Alkohol ab und nimmt das zurückbleibende Öl mit Äther auf. Die ätherische Lösung wird zur Entfernung von überschüssigem Jod mit verdünnter Natronlauge, dann mit Wasser durchgeschüttelt, mit entwässertem Natriumsulfat getrocknet, der Äther abdestilliert und das zurückbleibende braunrote Öl im Vakuum fraktioniert. Das 4-Jodveratrol geht bei einem Drucke von $12 \mathrm{~mm}$ von 150 bis $170^{\circ}$ als gelbes Öl über, das durch Abkühlen in einer Kältemischung oder Einführen eines Impf krystalles bei gewöhnlicher Temperatur vollständig erstarrt. Durch Umkrystallisieren aus verdünntem Methylalkohol erhält man farblose Nadeln, die den in der Literatur für 4-Jodveratrol angegebenen Schmelzpunkt von $35^{\circ}$ zeigen (Tassilly und Leroide 34 bis $35^{\circ}$ ). Die Ausbeute an reinem 4-Jodveratrol betrug $12 \mathrm{~g}$; sie konnte durch Verlängerung der Reaktionsdauer nicht verbessert werden.

\section{$3,4,3^{\prime}, 4^{\prime}$-Tetramethoxy -1, $1^{\prime}$ - biphenyl (Formel VIII).}

Gleiche Gewichtsteile 4-Jodveratrol und Kupferpulve werden in Kohlendioxydatmosphäre im Verlaufe einer halbe 
Stunde bis $260^{\circ}$ erhitzt und die Temperatur des Bades dann noch eine Viertelstunde auf der gleichen Höhe erhalten. Der erkalteten und gepulverten Schmelze entzieht man das Reaktionsprodukt entweder durch mehrmalige Extraktion mit Methylalkohol oder durch Sublimation im Kohlendioxydstrom. Es krystallisiert aus verdünntem Methylalkohol in farblosen seidenglänzenden Nädelchen vom Schmelzpunkt 130 bis $132^{\circ}$. $0 \cdot 1262 g$ Substanz: $0 \cdot 3262 g \mathrm{CO}_{2}, 0 \cdot 0780 g \mathrm{H}_{2} \mathrm{O}$.

In 100 Teilen:

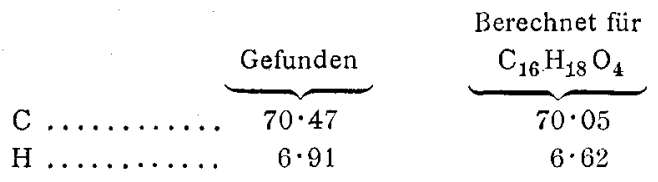

$3,4,3^{\prime}, 4^{\prime}$-Tetramethoxy-1, $1^{\prime}$ - biphenyl löst sich schon in der Kälte leicht in konzentrierter Schwefelsäure mit goldgelber Farbe, die bei gewöhnlicher Temperatur allmählich, bei gelindem Erwärmen schnell in Smaragdgrün übergeht. 